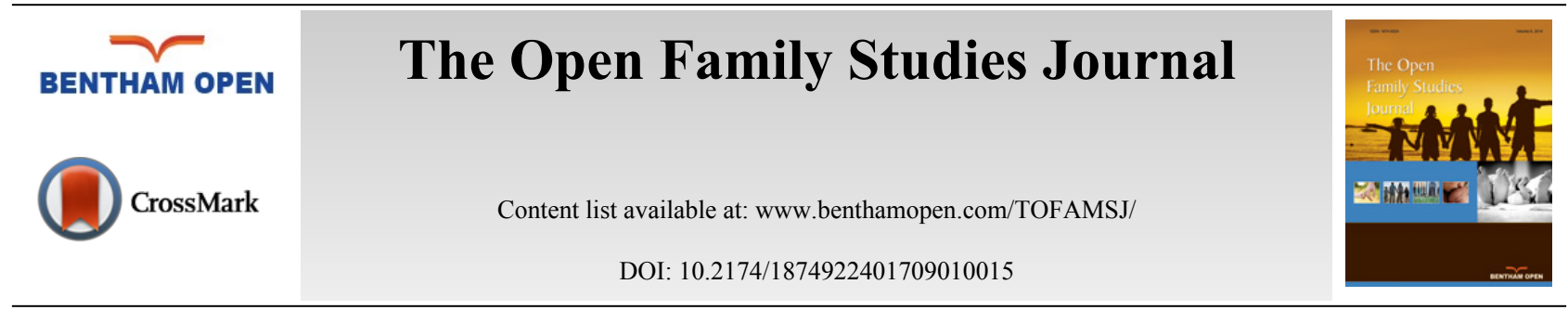

RESEARCH ARTICLE

\title{
An Application of Intervention Mapping as a Phased Approach in Developing a Family-centred Programme to Reduce Violence in the Family
}

Jill Ryan ${ }^{*}$ and Nicolette V. Roman

Child and Family Studies, Department of Social Work, University of the Western Cape, Bellville, Cape Town, 7353, South Africa

Received: October 22, 2016

Revised: March 15, 2017

Accepted: March 31, 2017

\section{Abstract:}

Aim:

To describe the application of intervention mapping in the development of a family-centred approach to reduce violence in the family.

\section{Methods:}

Development towards a family-centred approach is described, in which only the first three steps of the five intervention mapping steps have been detailed. These three steps are unpacked as phases. Phase I, a family violence needs assessment which includes a policy analysis. Phase II determines appropriate theoretical and practical approaches through systematic reviews and lastly, Phase III as a Delphi study.

\section{Results:}

Intervention mapping as a form of programme development is inclusive of all relevant stakeholders and allows diverse engagement with family-centred information through its structured development process.

\section{Conclusion:}

Family violence should be seen as a holistic phenomenon which affects all members of the family and future interventions must be mindful of this to address family violence effectively.

Keywords: Intervention mapping, Family-centred programme, Family violence, Future interventions, Delphi study, family-centred information.

\section{INTRODUCTION}

Human beings can only be understood and helped within the context of their intimate and powerful human systems, one of which is family [1]. Family is constructed and developed through its generations and has far-reaching effects on all its members [1]. Family members are shaped through mutual interaction and influence. These mutual interactions and influences create habitual ways of behaving and communication (the manner in problem-solving, arguments or discussions occur) and relationship patterns which govern these actions [2]. All of these mentioned activities create a family which promotes and provides care, stability, dependability and positive development as families are expected to provide [3]. However, such positive development can be disrupted through the occurrence of family violence whereby harm is purposefully inflicted by family members [4] resulting in a dysfunctional, entropic family system.

\footnotetext{
* Address correspondence to this author at the Child and Family Studies, Department of Social Work, University of the Western Cape, Bellville, Cape Town, 7353, South Africa; Tel: (+27) 219592970; E-mail: jillryan23@gmail.com
} 
Family violence refers to cohabiting persons who perform any act or omission that results in serious injury (physical, emotional harm or rights violation) to other members in the family [5]. Family violence characteristics further extend to include psychological aggression, coercion or intimidation to incite fear or intent to commit violent acts [6]. However family violence is usually divorced from the concept of family and is explored and understood in its various subsets as if separate, independently occurring phenomena with no shared qualities [6]. These subsets include i) intimate partner violence whereby the focus is on abuse occurring between current/former spouses or dating partners, ii) child abuse and maltreatment where the focus usually is abuse or neglect carried out by parent or caregiver on the child yet also abuse between siblings and lastly iii) elder abuse whereby the focus is on abuse between a caregiver or persons in a position of trust who inflict harm onto the elderly [7]. Recent findings have established that all subsets of family violence share an interconnected relationship and that all family members are affected by any act of violence occurring in the family, however few scholars have identified family violence as an integrated concept [6].

Globally, 275 million children are exposed to violence at home, with a strong correlation shown between violence against women and children and negative psychosocial challenges which span generations [8]. Youth who had witnessed violence at home were more likely to carry weapons, fight as well as threaten or injure others as opposed to youth who had not witnessed violence at home [9]. In the case of elder abuse, family members are noted as being the most likely perpetrators $[10,11]$.

Family violence, due to its pervasive nature and health implications, has shown to be a major public health concern $[12,13]$. For this reason, family violence has been addressed through health promotion programmes at various levels such as individual, school based and community [14]. Health promotion programmes have been proven to be effective when developed and implemented in a systematic manner in which effective planning and evaluation take place [15]. Intervention mapping proves to be such a framework, with systematic development and evaluation based on evidence based approaches delivered in structured steps [15]. Intervention mapping has five steps, 1) Specify the programme's goals into proximal programme objectives. In this stage, needs are identified; 2) Selection of theoretical and practical strategies; 3) Design the programme, 4) Implementation of the programme, and 5) Focus on anticipating process and effect evaluation [16]. To our knowledge, no intervention has been developed towards a holistic approach regarding family violence whereby all family members are included in the intervention.

The aim of this paper is to describe the application of intervention mapping in the development of a family-centred approach to reduce violence in the family. As only the development is described towards a family-centred approach, only the first three steps of the five intervention mapping steps have been detailed. These three steps will thus be referred to as phases. However steps four and five will be addressed under implications for programme implementation.

\section{PHASE I: FAMILY VIOLENCE NEEDS ASSESSMENT}

In phase I, a family violence needs assessment which can be done with families and practitioners, to identify the problem, which will create a comprehensive depiction of what issues are present in a family experiencing violence. Additionally it will also provide a scope for possible change regarding the target population's determinants of the issue $[16,17]$. This phase is divided into two stages which followed an explanatory sequential design in which to conduct a quantitative and qualitative needs assessment. Additionally a policy assessment can be added to this phase to holistically ascertain if policy and law currently provide a legislative environment which meets the needs of families experiencing violence at home but also provide a supportive setting for practitioners, who work with these families, to carry out their work effectively but are also provided adequate guidance through these legislative acts.

The first stage of the needs assessment relates to the quantitative aspect. The family functioning device is a beneficial instrument to use for the assessment as it provides definitive areas or domains that are tested for, provide areas of focus in the programme, specific to families and their functioning. With families, the family assessment device describes the structural and organizational properties of the family as well as the transaction patterns between family members. These indicators are used to determine healthy and unhealthy family functioning and are assessed according to the dimensions of family life $[18,19]$. The seven dimensions (scales) are tested in the assessment tool namely 1.) Problem Solving, 2) Communication, 3) Roles, 4) Affective responsiveness, 5) Affective involvement, 6) Behaviour control, and 7) General functioning [27]. The FAD, though first implemented in English, FAD has enjoyed successful implementation across cultures and translated in over 20 different languages [19, 20]. When approaching family practitioners, a shortened version of the family assessment device can be used, called the family functioning selfadministered questionnaire. This questionnaire was created to ascertain the core aspects of the family interpersonal functioning, an aim usually focused upon and addressed in family psycho-educational programmes [21, 22]. Though the 
McMaster Family Assessment Device (FAD) is notably popular in assessing how family members perceive the family environment and interaction, the family functioning (FF) questionnaire honed directly in on skills family members should have in order to help one another [21].

The qualitative aspect of this assessment relate to interviews with practitioners who work with families, regarding their experiences with families who experience violence at home as well as with these families as well as the families. This stage is done as the quantitative stage of the needs assessment only provides the descriptive perception of the practitioner experiences with families who experience violence at home as well as the experiences of the families. The interviews provide an opportunity for participants to elaborate on what should be included in the intervention.

\section{THE FAMILY POLICY ANALYSIS}

Family is rarely addressed within violence related policy as focus is placed on the victim/perpetrator and alignment of the justice system to this relationship. Yet the normalization of violence begins within the family and can be further exacerbated by community violence all of which leads to desensitization of violence as well as violence being seen as socially appropriate to resolve interpersonal conflict [23]. In order for the family to gain more focused attention within policy and to garner a more holistic response when faced with violence, attention needs to be directed at what is currently available to address violence occurring in the family.

Policy analysis is grounded within pragmatism as it creates, assesses and communicates policy-relevant information, in order to clarify and improve practical problems found to be complexly political, cultural, economic and ethical all at once [24].

\section{PHASE II: SELECTION OF THEORETICAL AND PRACTICAL STRATEGIES FOR INTERVENTION}

The aim of this phase would be to systematically review studies which describe strategies/models/methods/ approaches used for family-centred programmes regarding family violence. A systematic review is an optimal way to discover the best theoretical approaches for family violence and best help inform intervention formation with best practice, evidence-based approaches. Approaches based on theory optimise generalizability of results as well as ensure that the results obtained are not spurious [25]. Systematic reviews attempt to collate empirical evidence that fit prespecified eligibility criteria by using a methodology that can be reproduced, in order to minimise bias and increase validity and reliability of results [26]. Systematic reviews make use of hierarchies of evidence to assist in defining its eligibility criteria which ensures reliable and valid results. Hierarchies of evidence are scales used to grade research designs in order to limit risk of error and bias in order for only the best available evidence to be used to develop and implement health care interventions [27]. Through this rigorous process of the systematic review, only the most scientifically valid and reliable studies will be identified in order to best inform, by means of theoretical and evidence based strategies, development and design of an intervention programme for family violence.

\section{PHASE III: DESIGN THE PROGRAMME}

The Delphi method is implemented in order to engage various stakeholders regarding family violence in order to generate unanimity on the best possible strategy to address the phenomenon of family violence. The Delphi method entails a systematic engagement of experts who specialize in the field of interest being addressed, with the aim of reaching consensus and in generating an opinion on the topic of interest $[28,29]$. This is particularly important for intervention development [30]. Through this engagement, the scope as well as the various components of the programme is defined and various channels of delivering the intervention can be proposed.

\section{IMPLICATIONS}

This intervention minimises the misconception that family violence is mainly about the victim and perpetrator and emphasises the inclusivity of all the family members it affects. The intervention can be implemented cross-culturally and at any level, as the development process which includes engagement with diverse families and practitioners, international research done in various settings as well as engaging various stakeholders allowed for a diversified yet rich findings. This intervention can be implemented by organizations as well as government departments which work with families experiencing violence at home, with any family focused practitioner as well as trained facilitator to act as implementers.

The intervention should be piloted for efficacy before finalisation. The assessment items used in phase I can be used as evaluation tools to assess change within family functioning with one family group receiving family well-being 
sessions (focus areas defined through the development process) as well as a family conference meeting to collectively plan a way forward by implementing what was learnt with facilitator guidance, as compared to the group receiving family well-being sessions.

\section{LESSONS LEARNED}

The current approach proposed in this paper has currently been implemented as a PhD study, and as such important issues can be gleaned from this process. Intervention mapping is a form of programme development which is inclusive of all relevant stakeholders of whom the programme developed will ultimately benefit and be utilised by. The study design proposed by this paper is a way forward to create a comprehensive yet holistic intervention which is familycentred to reduce violence in the family (Fig. 1). However, family violence is an intricate topic, and when researched, proves to be quite challenging especially when attempting to engage families experiencing violence as well as interviewing the practitioners who assist them. Recruitment which was initially done for families proved to be quite challenging. Research within the field of violence has more defined ethics than any other sensitive topics [31] as research conducted could put both participant and researcher at risk if safety concerns are ignored [31]. To reduce harm and enhance benefit in accordance to ethics outlined nationally and internationally [31, 32], organizations which were already working with families experiencing violence were approached. Permission was sought from the various national offices to approach regional offices. Even with this permission, it was at the discretion of the individual social workers and counsellors of the organizations if they could assist or not.
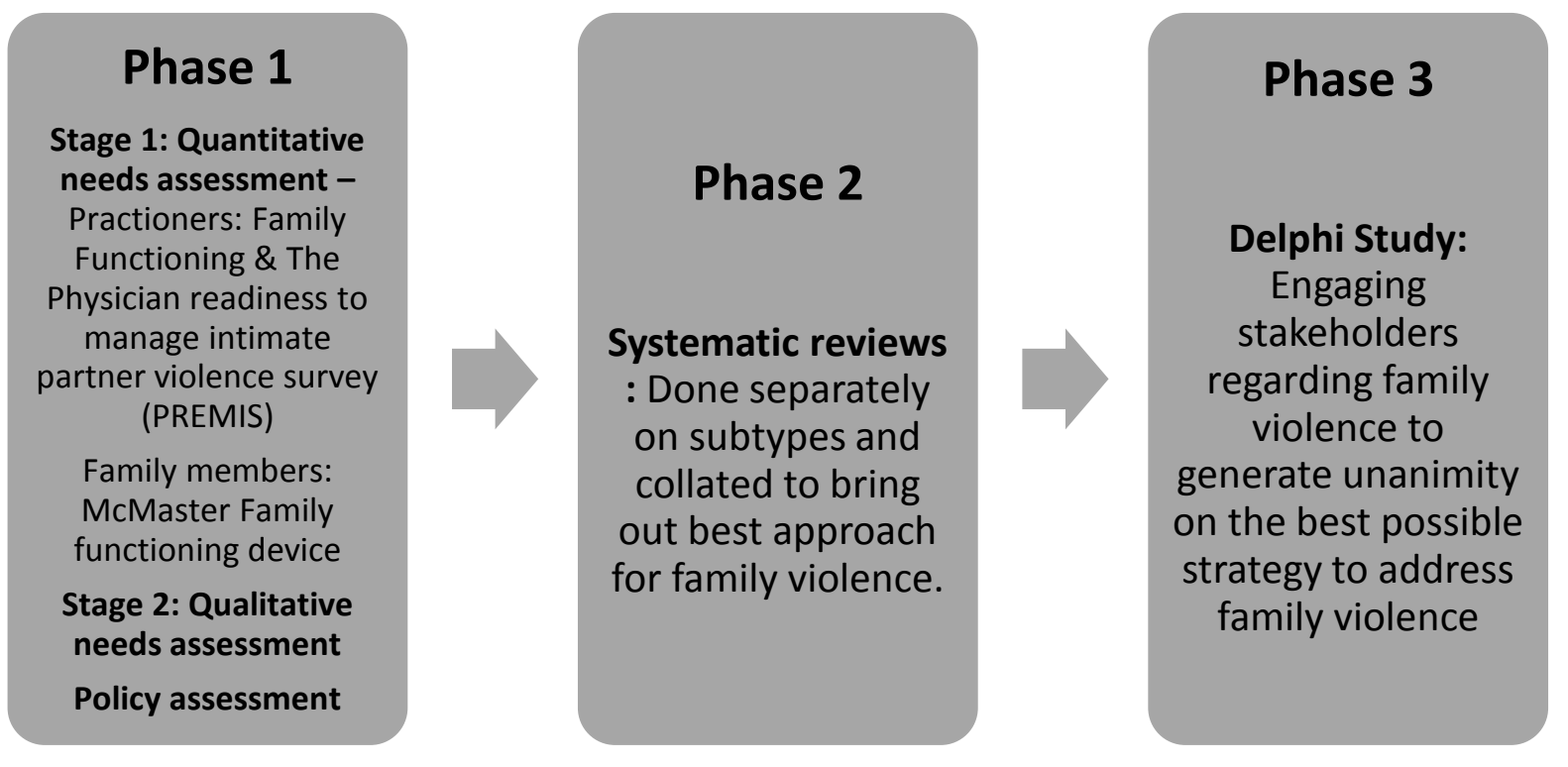

Fig. (1). Overveiw of study design.

With agreement reached invitation letters were sent to offices within the regional area inviting existing clients and their families to participate, after a year and a half only one participant was recruited. Even after much interaction, attending regional shelter forum meetings for further recruitment, and checking up with the organizations, it was either through non-response or lack of time on an already limited staff which resulted in non participation. Additionally, organizations contacted, who particularly worked with the elderly, claimed that their clients were not prepared to participate as the topic was a sensitive subject which they did not want to talk about. This scenario is quite common regarding elder abuse as underreporting was owed to a sense of protecting the family or a sense of shame or helplessness [33]. Organizations as gatekeepers to the community and the clients played a major role, not only in the initial family recruitment which organization resistance contributed much to challenges experienced. However when restrategized data collection took place, the organizations played a valuable role in networking. Re-strategizing of the data collection took place, as it was noticed that organization staff were more comfortable in relaying their own experiences and insight of families experiencing violence, than allowing access to their client base. For this reason only practitioners were consulted in the needs assessment phase with the addition of the policy analysis. But this too proved to be 
challenging as organizations present for the initial family recruitment withdrew due to fears that approaching staff to participate would add to the burden of already overworked, time-constrained, limited staff present at the organizations. However, all organizations approached confirmed that a family-centred intervention aimed at reducing family violence was needed.

\section{CONCLUSION}

The concept of family violence is shown to be quite disparate in its representation of all forms of abuse, as it is still dissected and addressed within its subsets (child abuse, elder abuse and intimate partner violence). It is hoped that this paper will bring to light the need of family violence being seen as a holistic phenomenon which affects all members of the family and should promote future interventions or approaches to address family violence in this manner on being cognizant of its effects on all family members experiencing the violence with its solution grounded in family centred strategies.

\section{CONFLICT OF INTEREST}

The authors confirm that this article content has no conflict of interest.

\section{ACKNOWLEDGEMENTS}

The authors would like to acknowledge the National Research Foundation (NRF) of South Africa for their funding of this PhD study.

\section{REFERENCES}

[1] Hartman A, Laird J. Family-centered social work practice. New York: Free Press 1983.

[2] Becvar DS, Becvar RJ. Family therapy: A systemic integration. In: Essex. England: Pearson Allyn and Bacon 2006.

[3] Amoateng AY, Richter M, Makiwane M, Rama S. Describing the structure and needs of families in South Africa: Towards the development of a national policy framework for families A report commissioned by the Department of Social Development. Pretoria: Child Youth and Family Development, Human Sciences Research Council 2004.

[4] Jouriles EN, McDonald R, Norwood WD, Ezell E. Issues and controversies in documenting the prevalence of children's exposure to domestic violence. In: Graham-Bermann SA, Edleson JL, Eds. Domestic violence in the lives of children: The future of research, intervention, and social policy. Washington, DC, US: American Psychological 2001; pp. 12-34.

[http://dx.doi.org/10.1037/10408-002]

[5] Wallace PH, Roberson C. 2015. Family violence: Legal, medical, and social perspectives. Routledge Aug 272015

[6] Tolan P, Gorman-Smith D, Henry D. Family violence. Annu Rev Psychol 2006; 57: 557-83. [http://dx.doi.org/10.1146/annurev.psych.57.102904.190110] [PMID: 16318607]

[7] McClennen J. Social Work and Family Violence: Theories Assessment, and Intervention. Springer Publishing Company 2010 ; pp. 132-3.

[8] UNICEF The state of the world's children 2008: Child survival. New York, USA: UNICEF 2007.

[9] Holborn L, Eddy G. First steps to healing the South African family. Johannesburg, South Africa: South African Institute of Race Relations 2011.

[10] Berkamn B. Handbook of Social Work in Health and Ageing. Oxford: Oxford University Press 2006. [http://dx.doi.org/10.1093/acprof:oso/9780195173727.001.0001]

[11] Soares Joaquim JF, Barros H, Torres-Gonzales F, et al. Abuse and health in Europe. Kaunas In: Kaunas, Lithuania: Lithuanian University of Health Sciences Press 2010; B84.

[12] WHO regional estimates of violence against women: Prevalence and health effects of intimate partner violence and non-partner sexual violence. Geneva: World Health Organization 2013.

[13] Devries KM, Mak JY, García-Moreno C, et al. Global health. The global prevalence of intimate partner violence against women. Science 2013; 340(6140): 1527-8.

[http://dx.doi.org/10.1126/science.1240937] [PMID: 23788730]

[14] Harvey A, Garcia-Moreno C, Butchart A. Primary prevention of intimate-partner violence and sexual violence Background paper for WHO expert meeting May 2-3 Geneva: World Health Organization, Department of Violence and Injuy Prevention and Disability 2007.

[15] Nyembezi A, Reddy P, Ruiter RA, van den Borne B, Sifunda S, Funani I. The application of intervention mapping in developing STI/HIV health education program for traditionally circumcised men in the Eastern Cape Province of South Africa. Europ Health Psycho 2016; 18(1): $22-9$.

[16] Kok G, Schaalma H, Ruiter RA, van Empelen P, Brug J. Intervention mapping: protocol for applying health psychology theory to prevention programmes. J Health Psychol 2004; 9(1): 85-98. [http://dx.doi.org/10.1177/1359105304038379] [PMID: 14683571] 
[17] Nutbeam D. Evaluating health promotion-progress, problems and solutions. Health Promot Int 1983; 13(1): 27-44.

[18] NB Epstein, LM Baldwin, DS Bishop. The McMaster family assessment device. J Marital Fam Ther 1983; 9(2): 171-80.

[19] Miller IW, Ryan CE, Keitner GI, Bishop DS, Epstein NB. The McMaster approach to families: Theory, assessment, treatment and research. J Fam Ther 2000; 22(2): 168-89.

[http://dx.doi.org/10.1111/1467-6427.00145]

[20] Ryan CE, Epstein NB, Keitner GI. Evaluating and treating families: The McMaster approach. Park Drive, UK: Taylor \& Francis 2005.

[21] Casacchia M, Roncone R. Italian families and family interventions. J Nerv Ment Dis 2014; 202(6): $487-97$. [http://dx.doi.org/10.1097/NMD.0000000000000149] [PMID: 24879572]

[22] Roncone R, Mazza M, Ussorio D, et al. The questionnaire of family functioning: A preliminary validation of a standardized instrument to evaluate psychoeducational family treatments. Community Ment Health J 2007; 43(6): 591-607. [http://dx.doi.org/10.1007/s10597-007-9093-8] [PMID: 17619149]

[23] Leoschut L. Running nowhere fast: Results of the 2008 National Youth Lifestyle study. Centre for Justice and Crime Prevention 2009.

[24] Dunn WN. Public policy analysis. Abingdan, UK: Routledge, 2015.

[25] Katz MH. Evaluating clinical and public health interventions: A practical guide to study design and statistics. Cambridge, UK: Cambridge University Press 2010. [http://dx.doi.org/10.1017/CBO9780511712074]

[26] Becker L, Oxman A. Overviews of reviews In: Higgins JPT, Green S, Eds Cochrane handbook for systematic reviews of interventions version 510 (updated March 2011), The Cochrane Collaboration. 2011. Available from: www.cochrane-handbook.org

[27] Evans D. Hierarchy of evidence: a framework for ranking evidence evaluating healthcare interventions. J Clin Nurs 2003; 12 (1): 77-84. [http://dx.doi.org/10.1046/j.1365-2702.2003.00662.x] [PMID: 12519253]

[28] Hasson F, Keeney S, McKenna H. Research guidelines for the Delphi survey technique. J Adv Nurs 2000; $32(4)$ : 1008-15. [PMID: 11095242]

[29] Hasson F, Keeney S. Enhancing rigour in the Delphi technique research. Technol Forecast Soc Change 2011; 78(9): 1695-704. [http://dx.doi.org/10.1016/j.techfore.2011.04.005]

[30] Skulmoski GJ, Hartman FT, Krahn J. The Delphi method for graduate research. J Inf Technol Educ 2007; 6: 1.

[31] Ellsberg M, Heise L. Researching violence against women: A practical guide for researchers and activists. Washington, DC, United States: World Health Organization, PATH 2005.

[32] Ethics in health research: Principles, processes and structures. 2nd ed. Pretoria, South Africa: Department of Health 2015.

[33] Kwiatkowski SM. Role of research on elder abuse: Introducing main issues. In: Jagielaska K, Łuksik JM, Pikuła NG, Eds. Violence against the elderly: Challenges-research-action, Toronto: European Association of Schools of Social work 2015; pp.9-16.

\section{(C) 2017 Ryan and Roman.}

This is an open access article distributed under the terms of the Creative Commons Attribution 4.0 International Public License (CC-BY 4.0), a copy of which is available at: https://creativecommons.org/licenses/by/4.0/legalcode. This license permits unrestricted use, distribution, and reproduction in any medium, provided the original author and source are credited. 